\title{
Incidence and Mortality trends of Atrial Fibrillation/Atrial Flutter in the United States 1990 to 2017
}

Augustin J. DeLago a,b, MD; Mohammed Essa ${ }^{\mathrm{a}, \mathrm{b}}$, MD, MBBS; Alireza Ghajar ${ }^{\mathrm{a}, \mathrm{b}}$, MD; Matthew Hammond-Haley $^{\mathrm{c}}$, MBBS; Arshi Parvez ${ }^{\mathrm{a}, \mathrm{b}}$ MBBS; Isaiah Nawaz ${ }^{\mathrm{a}}$, Joseph Shalhoub ${ }^{\mathrm{d}}$, BSc, MBBS, FHEA, PhD, MEd; Dominic C. Marshalle , MRCP MBBS; Saman Nazarian ${ }^{\mathrm{f}}$, MD, PhD; Hugh Calkins ${ }^{\mathrm{g}}$, MD; Justin D. Salciccioli ${ }^{\mathrm{a}, \mathrm{b}}$, MBBS, MA; Binu Philips ${ }^{\mathrm{b}, \mathrm{h}}$, MD

\section{Departments and Affiliations:}

${ }^{a}$ Department of Medicine, Mount Auburn Hospital, Cambridge, Massachusetts

${ }^{\mathrm{b}}$ Harvard Medical School, Boston, Massachusetts

${ }^{\mathrm{c}}$ National Heart and Lung Institute, Imperial College, London, UK

${ }^{\mathrm{d}}$ Imperial Vascular Unit, Imperial College Healthcare NHS Trust, London, UK and Academic Section of Vascular Surgery, Department of Surgery \& Cancer, Imperial College London, London, UK

${ }^{\mathrm{e}}$ British Heart Foundation Centre of Excellence, King's College London, London, UK

${ }^{\mathrm{f}}$ Department of Medicine, Division of Cardiology, Section of Cardiac Electrophysiology, Hospital of the University of Pennsylvania, Philadelphia, Pennsylvania

${ }^{\mathrm{g}}$ Department of Medicine, Division of Cardiology, Johns Hopkins Hospital, Baltimore, Maryland

${ }^{\mathrm{h}}$ Department of Medicine, Division of Cardiology, Mount Auburn Hospital, Cambridge, Massachusetts 02138

Running title: AF/AFL incidence and mortality trends in the US

Funding: No funding has been provided.

Disclosures: No disclosures to declare.

Corresponding Author: Binu Philips

Address for Correspondence: Binu Philips, MD

300 Mount Auburn Street, Suite 310 Cambridge, MA 02138

Phone: 617-497-156

Email: bphilips@mah.harvard.edu

Tweet: "Atrial Fibrillation/Flutter disease related incidence and mortality on rise across the United States."

Acknowledgements: We would like to express appreciation to Dr. Donald Cutlip for his time and valuable feedback. 


\section{Abstract}

Atrial fibrillation (AF) and flutter (AFL) are the most common clinically significant arrhythmias in older adults with an increasing disease burden due to an aging population. However, up-todate trends in disease burden and regional variation remain unknown. In an observational study utilizing the Global Burden of Disease (GBD) database, age-standardized mortality and incidence rates for AF overall and for each state in the United States (US) from 1990-2017 were determined. All analyses were stratified by sex. The relative change in age-standardized incidence rate (ASIR) and age-standardized death rate (ASDR) over the observation period were determined. Trends were analyzed using Joinpoint regression analysis. The mean ASIR per 100,000 population for men was $92(+/-8)$ and for women was $62(+/-5)$ in the US in 2017. The mean ASDR per 100,000 population for men was $5.8(+/-0.3)$ and for women was $4.4(+/-0.4)$. There were progressive increases in ASIR and ASDR in all but one state. The states with the greatest percentage change in incidence were New Hampshire $(+13.5 \%)$ and Idaho $(+16.0 \%)$ for men and women, respectively. The greatest change regarding mortality was seen in Mississippi $(+26.3 \%)$ for men and Oregon $(+53.8 \%)$ for women. In conclusion these findings provide updated evidence of increasing AF/AFL incidence and mortality on a national and regional level in the Unites States, with women experiencing greater increases in incidence and mortality rates. This study demonstrates that the public health burden related to AF in the United States is progressively worsening but disproportionately across states and among women.

Key Words: Atrial Fibrillation/Flutter, United States, Incidence, Mortality, Gender 


\section{Introduction}

Atrial fibrillation (AF) is a heart rhythm disorder characterized by rapid disorganized electrical activity originating in the atrium which is associated with increased morbidity and mortality ${ }^{1,2}$. The likelihood of developing AF is complex and influenced by biological factors, environmental factors, genetics, epigenetics, and social determinants ${ }^{3-8}$. Studies have shown that the prevalence and incidence of AF have been increasing in North America and Europe from the 1970s to the beginning of the $21^{\text {st }}$ century ${ }^{9}$. It is estimated that the prevalence of AF ranges from 2.7 to 6.1 million people in the United States (US) and this number is expected to increase to 12.1 million in $2030{ }^{10}$. The upsurge in AF is straining the US healthcare system. The net incremental cost of $\mathrm{AF}$ was shown to be $\$ 8705$ per patient and the national incremental cost was estimated to range between $\$ 6$ and $\$ 26$ billion based on prevalence data from 2004 to $2006^{11,12}$. Despite the growing public health challenge posed by AF, detailed up-to-date trends on the epidemiology of AF on a national and state level in the US remain limited. An accurate understanding of the current epidemiology of AF is crucial to the appropriate allocation of resources and therapies aimed at reducing the clinical and socioeconomic impact of the disease.

The objectives of this study were to determine current mortality and incidence rates for atrial fibrillation (AF)/flutter (AFL) overall and for each state in the United States across a defined time period using the Global Burden of Disease database.

\section{Methods}

For this observational analysis of AF incidence and mortality, data collected for the Global Burden of Disease (GBD) study was utilized. The GBD methodology has been published previously and reports global results for health loss related to specific diseases: deaths and death rates, years of life lost due to premature mortality, prevalence and incidence. The study data are estimated annually and each round of results are internally consistent (i.e. cause-specific mortality estimates match all-cause mortality estimates). The data are made available publicly (http://ghdx.healthdata.org/gbd-results-tool).

In the GBD methodology, $\mathrm{AF}$ was defined as a diagnosis of $\mathrm{AF}$ or atrial flutter by $\mathrm{ECG}$ finding. GBD study attributes each death to a single underlying cause that began the series of events leading to death, in accordance with International Classification of Diseases (ICD)-10 principles. To estimate incidence and mortality of AF/flutter, GBD used a systematic review strategy to identify claims data for both in-patient and out-patient visits in the US. To address 
changes in coding practices of AF, GBD uses an approach which combines disease model (ie. 'DisMod-MR') tool and cause of death ensemble model (ie. 'CODEm') to estimate deaths from $\mathrm{AF}$ and AFL. These tools are used to pool disparate information on incidence, prevalence, and mortality for varying age groupings and from varying age groupings from data sources that use varying methods. This combined approach allows for more accurate estimate of the number of deaths attributable to AF/flutter. We extracted, incidence and mortality estimates for AF between 1990 and 2017 across 51 US states were extracted from the online data repository.

Age-standardized incidence and mortality data per 100,000 population categorized by state, sex, and year were extracted from the GBD Results Tool web-based system. For all agestandardized rates, GBD uses a standard population calculated as the non-weighted average across all countries of the percentage of the population in each five-year age group for the years 2010 to 2035 from the United Nations Population Division's World Population Prospects (2012 revision) (GBD 2012, United Nations Department of Economics and Social Affairs Population Division). We computed relative change over the observation period by computing the difference between the start and end age-standardized incidence rates (ASIRs) and age-standardized death rates (ASDRs) for each sex in each country.

Trends were assessed using Joinpoint software (Version 4.5.0.1) provided by the United States National Cancer Institute Surveillance Research Program (28). Joinpoint regression analysis assesses changes in linear slope for mortality trends over time, as described previously (28). Briefly, it assesses the overall trends in mortality, initially with no Joinpoints, and tests for significant changes in the model with sequential addition of points where there is significant change in the slope of the line. The model also computes an estimated annual percentage change (EAPC) for each trend by fitting a regression line to the natural logarithm of the rates. The loglinear transformation allows us to approximation of normal distributions and by estimating the annual percentage change, it was possible to assess change in mortality trend at a constant percent per year. EAPCs are reported with 95\% Confidence Intervals (CIs). This method of statistical analysis has been previously utilized by Hartley et.al ${ }^{13}$.

\section{Results}

There were a total of 51 states or territories in the US each with 28 years of available data for analysis. Sex-specific, age-standardized incidence, and death per 100,000 population from 
Atrial fibrillation / Atrial flutter across 51 US states between 1990 and 2017 are shown in Supplementary Figures 1 and 2. There were no missing data elements. The mean 2017 incidence rate per 100,000 population for AF for men was 92 (+/-8) and the mean 2017 ASIR for women was $62(+/-5)$. For men, the greatest incidence rates in 2017 were observed in Massachusetts (105 per 100,000 population), New Hampshire (105 per 100,000 population), Maine (104 per 100,000 population) and Connecticut (104 per 100,000 population). For women, the greatest incidence rates were observed in Ohio (69 per 100,000 population), West Virginia (69 per 100,000 population), Louisiana (68 per 100,000 population), and Delaware (68 per 100,000 population). The 2017 incidence rates for each state are represented graphically in Table 1A.

The mean 2017 AF ASDR per 100,000 population for men was $5.8(+/-0.3)$ and the mean 2017 death rate for women was 4.4 (+/-0.4). For men, the greatest death rates were observed in New Hampshire (6.6 per 100,000 population), Maine (6.5 per 100,000 population), Montana (6.4 per 100,000 population), and Alaska (6.3 per 100,000 population). For women, the greatest death rates were observed in Oregon .7 per 100,000 population), Alaska (5.5 per 100,000 population), Montana (5.4 per 100,000 population), and Utah (5.1 per 100,000 population). The 2017 death rates for each state are represented graphically in Table 1B.

Across the 28-year study period, there was, overall, an increasing trend in the incidence of AF across the majority of US states. Among men, all states except District of Columbia ($1.7 \%$ change in men and a $-1.9 \%$ in women) show increase in the incidence rate of AF/AFL between 1990 and 2017. The greatest incidence rates for men in 2017 were clustered in the New England region. However, the states that showed the greatest percentage change in incidence for men were scattered throughout the Northeast, West coast, and South, with the top five being New Hampshire (+13.5\%), California (+13.3\%), Massachusetts $(+13.0 \%)$, and Maine $(+12.4 \%)$. Among females, all states except District of Columbia show an increase in the incidence rate of AF/AFL between 1990 to 2017. For women, the states with the greatest incidence rates and percentage change in incidence did not appear geographically clustered; with Idaho $(+16.0 \%)$, California (+15.5\%), Maine (+15.3\%), and Washington (+14.6\%) showing the highest increase. A summary of overall incidence rate percentage changes for both men and women are shown in Figure 1.

Apart from Alaska, where there was overall decrease in death rate in men (-8.4\%) and in women $(-2.9 \%)$, there were overall increasing death rates in both men and women in the majority 
of states. The greatest percentage increases were, for men: Mississippi $(+26.3 \%)$, Oklahoma $(+26.3 \%)$, Idaho $(+24.9 \%)$, and Kentucky (+23.4\%); and for women: Oregon $(+53.8 \%)$, Montana (+47.2\%), Utah (+44.8\%), and Nebraska (+40.0\%). A summary of overall mortality rate percentage changes for both men and women are shown in Figure 2.

Supplementary Figures 1 and 2 demonstrate an overall increasing trend in incidence and death rate for most of the US states. Supplementary tables 1A and 1B present the results of a Joinpoint analyses for ASIRs for AF between 1990 to 2017 in men and women, respectively. Supplementary Tables 2A and 2B present the results of Joinpoint analyses for ASDRs. In each of these tables, EAPCs in rates for periods covered by each trend are reported. Significant trend changes in ASIRs and ASDRs are reported. There are significant increasing trends in AF incidence in most states for men and women. Lastly, an inflection point in 2001 is notable for increased incidence and mortality rates for both genders across most states.

\section{Discussion}

This study reports recent data relating to the epidemiology of atrial fibrillation (AF)/flutter (AFL) on a national and state level in the United States over a 28-year period. All regions of the US, apart from the District of Columbia, experienced an increase in AF incidence rates. The Northeast region was notable for having the highest incidence rates amongst men. While current incidence rates among states were higher for men than women, women had greater increases in incidence rates over the study period. In contrast to prior studies, our study showed a significant increase in mortality rates with the exception of the District of Columbia and Alaska. Furthermore, while overall mortality rates were higher in men, women had a significantly greater increase in mortality across most states over the study period. These data suggest that the public health burden related to AF in the US is progressively worsening, particularly since 2001, but disproportionately between states.

Incidence rates for AF in the US are expected to increase because of the aging US population and increased surveillance. However, studies from Europe and North America show discrepancy in the trend in AF incidence rates ${ }^{1415,16}$. The world-wide incidence of AF in 1990 was 60.7 per 100,000 person-years in males and 43.8 in females. In 2010, the world-wide incidence had increased to 77.5 per person-years in males and 59.5 in females ${ }^{17}$. Fifty year follow up of the Framingham Heart Study participants showed age-adjusted incidence was 3.7 and 2.5 per 1000 person-years among men and women, respectively, in the early time period of 
the study compared to 13.4 and 8.5 per 1000 person years among men and women respectively in the most recent time period ${ }^{18}$.

Prior studies have demonstrated that AF is associated with increased mortality, but survival has been trending upward over time. Globally, the mortality associated with AF has increased 2-fold in women and 1.9-fold in men ${ }^{17}$. In Medicare beneficiaries over 65 years of age, the age and sex-adjusted mortality at 30 days and 1 year was $11 \%$ and $25 \%$, respectively, in $2007^{15}$. In a community-based cohort of new-onset AF, the most common causes of death within 4 months of AF diagnosis were coronary artery disease (22\%), heart failure (14\%), and ischemic stroke (10\%) and beyond 4 months were heart failure (16\%), coronary artery disease (15\%), and ischemic stroke $(7 \%)^{19}$. In 1998, the Framingham Heart Study established that AF was associated with an increased risk of death in males (OR 1.5; 95\% CI 1.2-1.8) and females (OR 1.9; 95\% CI 1.5-2.2) ${ }^{1}$, Nonetheless, long term follow-up of Framingham Heart Study participants showed a $25 \%$ decrease over time in multivariable-adjusted mortality following AF onset when comparing the first to final time periods of the study ${ }^{18}$. This study also showed a $73.5 \%$ decline in stroke risk after AF onset when comparing the first to final time periods of the study. Similarly, a long-term Danish study showed a decrease in population-adjusted mortality from the first to last 5 year period by $20 \%$ in men and $18 \%$ in women ${ }^{20}$. The same researchers reported an improving trend in stroke mortality from 1980 to $2002^{21}$. On the other hand, the 2000-2010 community study of Olmstead County, MN showed that age- and sex-adjusted mortality rates did not change over time ${ }^{16}$. In contrast, our study showed a significant increase in mortality rates from 1990 to 2017. The exceptions were the District of Columbia for females and Alaska for both genders. The reason for the discrepancy in mortality trends in relation to AF is unclear and outside the scope of this study. This may, in part, be due to the underutilization of anticoagulation in $\mathrm{AF}^{22,23}$. In the US National Cardiovascular Data Registry of outpatients with $\mathrm{AF}$, less than one half of high-risk patients (defined as those with CHA2DS2-VASc score $\geq 4$ ) were receiving anticoagulation ${ }^{23}$. It has been shown that AF patients not treated with anticoagulation had 2.1-fold increased risk of recurrent stroke and 2.4-fold increase risk of recurrent severe stroke ${ }^{24}$.

Additionally, it is known that genetics confer survival advantage to females over males for most disease states except autoimmune diseases ${ }^{25,26}$. While mortality related to AF was higher across all states for men, in general women had significantly greater increases in mortality 
over the study period compared to men. The greatest percentage increase in mortality for men was in Mississippi which had 26\% increase in mortality from 1990 to 2017, while 16 states had greater than $30 \%$ increases in mortality for women. In fact, Oregon had a 54\% increase in AFrelated mortality for women. While there were no regional patterns for mortality among men, among women the greatest percentage increases in mortality rates were in the West and Mountain West and included Oregon Montana, Utah, and Nebraska.

Unlike prior studies, this study for the first time shows an inflection point in 2001 at which time there was a significant increase in the trend for both AF incidence and mortality in both genders across most states. The explanation for this finding would be speculative and outside the scope of this study. Nevertheless, this key finding has important public health implications and underscores the necessity of understanding the current epidemiology of AF.

Several limitations should be considered when interpreting the results of this study. First this is an observational study subject to limitations and biases of any observational study. Second, the GBD dataset did not distinguish between AF and AFL, or patterns of AF - such as paroxysmal or persistent - and therefore analysis could not be performed on these arrhythmia subsets. Third, the GBD database does not distinguish between clinical relevant or symptomatic cases and subclinical AF/AFL. Fourth, this database primarily relies upon hospital administrative data across various regional geographic regions, which stem from varying practices and quality care across hospitals

In conclusion, AF incidence and mortality rates are increasing across all states in the US, with the exceptions of the District of Columbia and Alaska. The upward trends have accelerated for most states since 2001 and women have experienced greater increases in incidence and mortality rates. This study demonstrates that the public health burden related to atrial fibrillation in the United States is progressively worsening particularly since 2001 but disproportionately across states and among women.

The burden of AF is worsening in the United States disproportionately among states and women and is an ever-increasing public health challenge 


\section{References}

1. Benjamin EJ, Wolf PA, D'Agostino RB, Silbershatz H, Kannel WB, Levy D. Impact of atrial fibrillation on the risk of death: the Framingham Heart Study. Circulation 1998;98:946-952.

2. Kannel WB, Wolf PA, Benjamin EJ, Levy D. Prevalence, incidence, prognosis, and predisposing conditions for atrial fibrillation: population-based estimates. Am J Cardiol 1998;82:2n-9n.

3. Benjamin EJ, Levy D, Vaziri SM, D'Agostino RB, Belanger AJ, Wolf PA. Independent risk factors for atrial fibrillation in a population-based cohort. The Framingham Heart Study. Jama 1994;271:840-844.

4. Psaty BM, Manolio TA, Kuller LH, Kronmal RA, Cushman M, Fried LP, White R, Furberg CD, Rautaharju PM. Incidence of and risk factors for atrial fibrillation in older adults. Circulation 1997;96:2455-2461.

5. Vaziri SM, Larson MG, Benjamin EJ, Levy D. Echocardiographic predictors of nonrheumatic atrial fibrillation. The Framingham Heart Study. Circulation 1994;89:724-730.

6. Aviles RJ, Martin DO, Apperson-Hansen C, Houghtaling PL, Rautaharju P, Kronmal RA, Tracy RP, Van Wagoner DR, Psaty BM, Lauer MS, Chung MK. Inflammation as a risk factor for atrial fibrillation. Circulation 2003;108:3006-3010.

7. Ko D, Benson MD, Ngo D, Yang Q, Larson MG, Wang TJ, Trinquart L, McManus DD, Lubitz SA, Ellinor PT, Vasan RS, Gerszten RE, Benjamin EJ, Lin H. Proteomics Profiling and Risk of New-Onset Atrial Fibrillation: Framingham Heart Study. J Am Heart Assoc 2019;8:e010976. 8. Weng LC, Preis SR, Hulme OL, Larson MG, Choi SH, Wang B, Trinquart L, McManus DD, Staerk L, Lin H, Lunetta KL, Ellinor PT, Benjamin EJ, Lubitz SA. Genetic Predisposition, Clinical Risk Factor Burden, and Lifetime Risk of Atrial Fibrillation. Circulation 2018;137:1027-1038.

9. Guez D, Boroumand G, Ruggiero NJ, Mehrotra P, Halpern EJ. Automated and Manual Measurements of the Aortic Annulus with ECG-Gated Cardiac CT Angiography Prior to 
Transcatheter Aortic Valve Replacement: Comparison with 3D-Transesophageal Echocardiography. Academic radiology 2017;24:587-593.

10. Colilla S, Crow A, Petkun W, Singer DE, Simon T, Liu X. Estimates of current and future incidence and prevalence of atrial fibrillation in the U.S. adult population. Am J Cardiol 2013;112:1142-1147.

11. Mehran R, Claessen BE, Godino C, Dangas GD, Obunai K, Kanwal S, Carlino M, Henriques JP, Di Mario C, Kim YH, Park SJ, Stone GW, Leon MB, Moses JW, Colombo A. Long-term outcome of percutaneous coronary intervention for chronic total occlusions. JACC CardiovasC Interv 2011;4:952-961.

12. Kim MH, Johnston SS, Chu BC, Dalal MR, Schulman KL. Estimation of total incremental health care costs in patients with atrial fibrillation in the United States. Circ Cardiovasc Qual Outcomes 2011;4:313-320.

13. Hartley A, Marshall DC, Salciccioli JD, Sikkel MB, Maruthappu M, Shalhoub J. Trends in Mortality From Ischemic Heart Disease and Cerebrovascular Disease in Europe: 1980 to 2009. Circulation 2016;133:1916-1926.

14. Miyasaka Y, Barnes ME, Gersh BJ, Cha SS, Bailey KR, Abhayaratna WP, Seward JB, Tsang TSM. Secular Trends in Incidence of Atrial Fibrillation in Olmsted County, Minnesota, 1980 to 2000, and Implications on the Projections for Future Prevalence. Circulation $2006 ; 114: 119-125$.

15. Piccini JP, Sinner MF, Greiner MA, Hammill BG, Fontes JD, Daubert JP, Ellinor PT, Hernandez AF, Walkey AJ, Heckbert SR, Benjamin EJ, Curtis LH. Outcomes of Medicare beneficiaries undergoing catheter ablation for atrial fibrillation. Circulation 2012;126:2200-2207. 16. Chamberlain AM, Gersh BJ, Alonso A, Chen LY, Berardi C, Manemann SM, Killian JM, Weston SA, Roger VL. Decade-long trends in atrial fibrillation incidence and survival: a community study. Am J Med 2015;128:260-267.e261. 
17. Chugh SS, Havmoeller R, Narayanan K, Singh D, Rienstra M, Benjamin EJ, Gillum RF, Kim YH, McAnulty JH, Jr., Zheng ZJ, Forouzanfar MH, Naghavi M, Mensah GA, Ezzati M, Murray CJ. Worldwide epidemiology of atrial fibrillation: a Global Burden of Disease 2010 Study. Circulation 2014;129:837-847.

18. Schnabel RB, Yin X, Gona P, Larson MG, Beiser AS, McManus DD, Newton-Cheh C, Lubitz SA, Magnani JW, Ellinor PT, Seshadri S, Wolf PA, Vasan RS, Benjamin EJ, Levy D. 50 year trends in atrial fibrillation prevalence, incidence, risk factors, and mortality in the Framingham Heart Study: a cohort study. Lancet 2015;386:154-162.

19. Miyasaka Y, Barnes ME, Bailey KR, Cha SS, Gersh BJ, Seward JB, Tsang TS. Mortality trends in patients diagnosed with first atrial fibrillation: a 21 -year community-based study. J Am Coll Cardiol 2007;49:986-992.

20. Frost L, Vestergaard $P$, Mosekilde L, Mortensen LS. Trends in incidence and mortality in the hospital diagnosis of atrial fibrillation or flutter in Denmark, 1980-1999. Int J Cardiol 2005;103:78-84.

21. Frost L, Andersen LV, Vestergaard P, Husted S, Mortensen LS. Trend in mortality after stroke with atrial fibrillation. Am J Med 2007;120:47-53.

22. Baczek VL, Chen WT, Kluger J, Coleman Cl. Predictors of warfarin use in atrial fibrillation in the United States: a systematic review and meta-analysis. BMC Fam Pract 2012;13:5.

23. Hsu JC, Maddox TM, Kennedy KF, Katz DF, Marzec LN, Lubitz SA, Gehi AK, Turakhia MP, Marcus GM. Oral Anticoagulant Therapy Prescription in Patients With Atrial Fibrillation Across the Spectrum of Stroke Risk: Insights From the NCDR PINNACLE Registry. JAMA Cardiol 2016;1:55-62.

24. Penado S, Cano M, Acha O, Hernández JL, Riancho JA. Atrial fibrillation as a risk factor for stroke recurrence. Am J Med 2003;114:206-210. 
25. Spolarics Z, Peña G, Qin Y, Donnelly RJ, Livingston DH. Inherent X-Linked Genetic Variability and Cellular Mosaicism Unique to Females Contribute to Sex-Related Differences in the Innate Immune Response. Front Immunol 2017;8:1455-1455.

26. Marais GAB, Gaillard J-M, Vieira C, Plotton I, Sanlaville D, Gueyffier F, Lemaitre J-F. Sex gap in aging and longevity: can sex chromosomes play a role? Biology of Sex Differences 2018;9:33. 


\section{Figures/Tables}

Table 1A: Age-standardized incidence rate in 2017 across US states for men and women.

Table 1B: Age-standardized death rate in 2017 across US states for men and women.

Figure 1: Percentage change in incidence rate between 1990 and 2017 from Atrial Fibrillation / Atrial Flutter across US states for men (A) and women (B).

Figure 2: Percentage change in death rate between 1990 and 2017 from Atrial Fibrillation / Atrial Flutter across US states for men (A) and women (B).

Supplementary table 1: Jointpoint analysis. Sex specific, age-standardized incidence rates 1990-2017 from Atrial Fibrillation / Atrial Flutter across US states for men (A) and women (B). Supplementary Table 2: Jointpoint analysis. Sex specific, age-standardized death rates 19902017 from Atrial Fibrillation / Atrial Flutter across US states for men (A) and women (B). Supplementary Figure 1: Sex-specific, age-standardized incidence per 100,000 population from Atrial fibrillation / Atrial flutter across 51 US states between 1990 and 2017. Squares represent men and circles represent women.

Supplementary Figure 2: Sex-specific, age-standardized death per 100,000 population from Atrial fibrillation / Atrial flutter across 51 US states between 1990 and 2017. Squares represent men and circles represent women. 